This is the pre-peer reviewed version of the following article:

Bellacanzone C., Roscini C., del Carmen Ruiz Delgado M., Ponce Ortiz R., Ruiz-Molina D.. Sonochemical Synthesis of Optically Tuneable Conjugated Polymer Nanoparticles. Particle and Particle Systems Characterization, (2018). 35. 1700322: - . 10.1002/ppsc. 201700322 ,

which has been published in final form at https://dx.doi.org/10.1002/ppsc. 201700322. This article may be used for non-commercial purposes in accordance with Wiley Terms and Conditions for Use of Self-Archived Versions. 
DOI: $10.1002 /(($ please add manuscript number $))$

Article type: Communication

\section{Sonochemical synthesis of optically tunable conjugated polymer nanoparticles}

Christian Bellacanzone, Claudio Roscini, * Maria Carmen Ruiz Delgado, Rocio Ponce Ortiz, Daniel Ruiz-Molina*

C. Bellacanzone, C. Roscini, D. Ruiz-Molina

Catalan Institute of Nanoscience and Nanotechnology (ICN2), CSIC and The Barcelona Institute of Science and Technology (BIST), Campus UAB, Bellaterra, 08193, Spain.

e-mail: daniel.ruiz@icn2.cat

M. C. Ruiz Delgado, R. Ponce Ortiz

University of Malaga, Department of Physical Chemistry, E-29071 Malaga, Spain.

Keywords: semiconducting polymers, fluorescent nanoparticles, sonochemistry, nanoemulsions, MEH-PPV

\section{Abstract.}

The development of novel and simple methodologies for the obtaining of semiconductive polymer nanoparticles with fine-tuned optical properties represents nowadays a challenging research area as it involves a simultaneous chemical modification and nanostructuration of the polymer. Here, starting from poly[2-methoxy-5-(2-ethylhexyloxy)-1,4-phenylenevinylene], we achieved this objective with the one-pot synthesis of oligomers with tunable conjugation length and their nanostructuration, employing a miniemulsion method. Ultrasound irradiation of heterogeneous mixtures led to the formation of hypochlorous acid that disrupts the electronic conjugation through polymer chain cleavage. Moreover, control over the degree of the electronic conjugation of the oligomers, and therefore of the optical properties, was achieved simply by varying the polymer concentration of the initial solution. Finally, the presence of surfactants during the sonication allowed for the formation of nanoparticles with progressive spectral shift of the main absorption (from $\lambda_{\max }=476 \mathrm{~nm}$ to $306 \mathrm{~nm}$ ) and emission bands (from $\lambda_{\max }=597 \mathrm{~nm}$ to $481 \mathrm{~nm}$ ). The integration of CNPs into polymeric matrices yielded self-standing and flexible fluorescent films. 
Given their high brightness, photostability and biocompatibility, conducting polymer nanoparticles (CNPs) have been proposed for different applications, from water processable inks for device fabrication (photovoltaic ${ }^{[1]}$ and electrochromic ${ }^{[2]}$ cells and OLEDs ${ }^{[3]}$ ) to nontoxic fluorescent biological labels, ${ }^{[4]}$ or even to induce photophysical processes at the nanoscale (e.g. photoinduced electron ${ }^{[5]}$ or energy transfer ${ }^{[6]}$ ). Though, to fully exploit the limitless possibilities of these NPs, further basic research is still required. One of such areas is the development of novel and simple methodologies for the obtaining of semiconductive nanoparticles with fine-tuned optical (especially emission) properties, ${ }^{[4]}$ due to its implicit technological relevance and the applications that can be derived from there. So far, spectral emission tuning of bulk semiconductive polymers has raised lots of interest and has been reported through encapsulation and confinement in nanoporous silica $\mathrm{NPs}^{[7]}$ systematic variation of solvent and polymer concentration ${ }^{[8]}$ or after post-fabrication annealing/cooling or pressure/mechanical cycles. ${ }^{[9-11]}$ Alternative chemical strategies include polymer modification with different core units ${ }^{[12]} /$ side substituents, ${ }^{[13]}$ or the controlled synthesis of oligomeric species differing on the number of repetitive monomers (and therefore conjugation length). ${ }^{[14]}$ Only very recently the synthesis of nanoparticles with tunable optical properties has been partially achieved through the synthesis of polymer dots with packing-dependent emission. ${ }^{[4]}$ Therefore, there is a need to find novel approaches for the simultaneous spectral tuning and nanostructuration of semiconductive polymers.

Sonication in chloroform/water mixtures can be an excellent technique to achieve this target. First, emulsification induced by ultrasonic radiation is a process broadly used for the formation of nanoparticles. Second, sonication of chloroform/water mixture is known to generate reactive species (see Supporting Information, Scheme S1) such as chlorine radicals 
$\left(\mathrm{Cl}, \mathrm{CHCl}_{2}, \mathrm{CCl}_{3}\right){ }^{[15]}$ and hypochlorous acid $(\mathrm{HOCl}),{ }^{[16]}$ which can be used to systematically cleave conjugated species, and therefore to modify the final optical properties. Indeed, chlorine radicals obtained by different means such as daylight, ${ }^{[17]} \gamma$-radiations ${ }^{[18]}$ or thermal treatment, ${ }^{[19-20]}$ have already been shown to tune the properties of poly[2-methoxy-5-(2ethylhexyloxy)-1,4-phenylenevinylene] (MEH-PPV) upon polymer fragmentation, ${ }^{\text {[17-20] }}$ whereas $\mathrm{HOCl}$ acts as strong oxidative species cleaving linear conjugated systems. ${ }^{[21-23]}$ Therefore, herein we hypothesize that sonication of the semiconductive polymer solutions of different concentrations is expected to induce different fragmentation degrees, resulting in the formation of nanoparticles with tunable optical properties. A schematic representation of this process is shown in Figure 1a. As a proof-of-concept to validate our approach, herein we propose the use of MEH-PPV, because of its excellent luminescence $\left(\mathrm{QY}^{\text {toluene }}=0.34\right){ }^{[24]}$ semiconducting $^{[25]}$ and non-linear optical ${ }^{[26]}$ properties.

a)

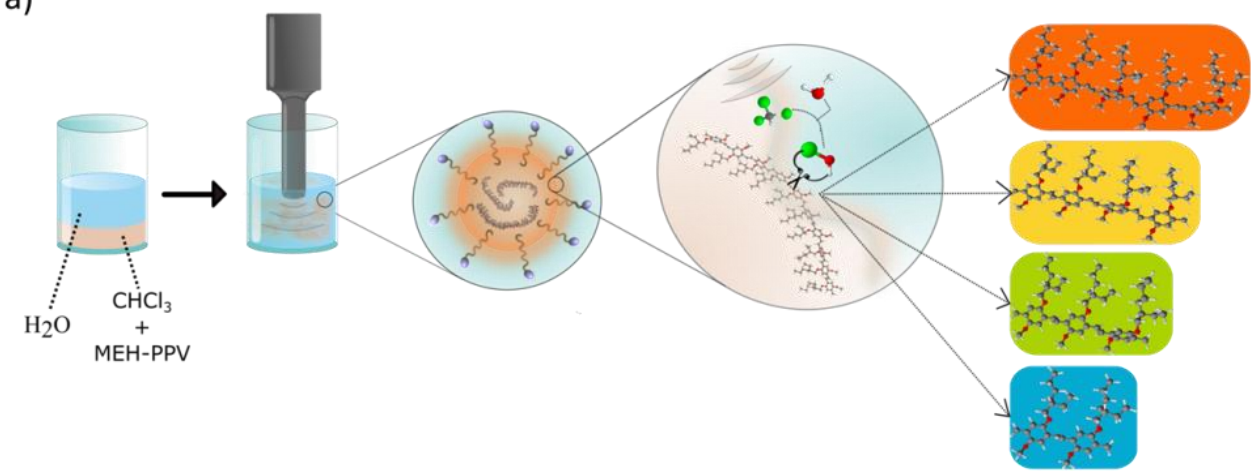

b)

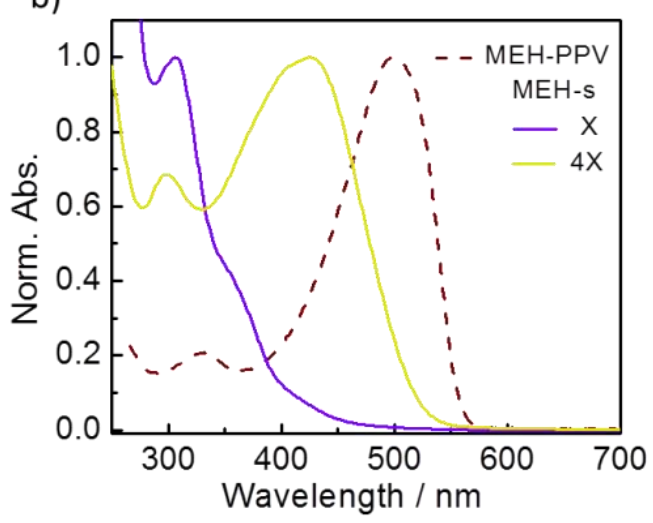

c)

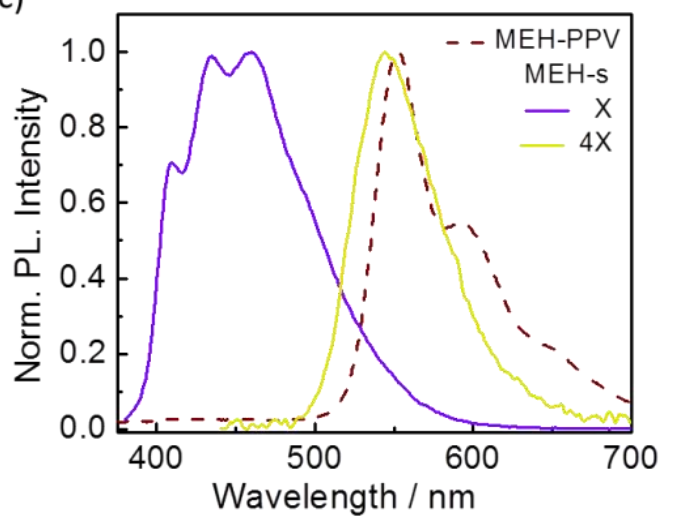


Figure 1. a) scheme of the sonication process of a mixture made of water and a MEH-PPV chloroform solution which produces different fragmentation units with tunable optical properties depending on the initial polymer concentration; $b$ ) absorption and $c$ ) emission spectra of untreated $\left(\lambda_{\max }{ }^{\text {abs }}=500 \mathrm{~nm}\right.$ and $\lambda_{\max }{ }^{\text {emis }}=552 \mathrm{~nm}$, dashed lines) and sonicated MEH-PPV solutions (MEH-s $4 X, \lambda_{\max }^{\text {abs }}=425 \mathrm{~nm}$ and $\lambda_{\text {max }}{ }^{\text {emis }}=544 \mathrm{~nm}$, purple; MEH-s X, $\lambda_{\max }{ }^{\text {abs }}=306 \mathrm{~nm}$ and $\lambda_{\max }{ }^{\text {emis }}=435$ and $460 \mathrm{~nm}$, yellow), recorded in $\mathrm{CHCl}_{3}$.

To demonstrate the validity of our approach, two MEH-PPV chloroform solutions, 2 $\mathrm{mM}$ (hereafter named as MEH-s X) and $8 \mathrm{mM}$ (MEH-s 4X), were sonicated in Milli-Q ${ }^{\circledR}$ water (for more details on the synthesis see the Supporting Information), at this stage without any surfactant, to avoid possible side reactions and allow an easier isolation of the formed products. Spectroscopic UV-Vis characterization of the extracted organic phases after sonication (MEH-s) showed a progressive hypsochromic shift of the main absorption band, from $\lambda_{\max }=500 \mathrm{~nm}$ for the parent untreated polymer to 425 and $306 \mathrm{~nm}$, for MEH-s $4 \mathrm{X}$ and MEH-s X samples, respectively (Figure 1b). Similar results were obtained for the fluorescence measurements (Figure 1c). A blue-shift of the emission band from $\lambda_{\max }=552$ $\mathrm{nm}$ of the untreated polymer to 544 (MEH-s 4X) and $435 \mathrm{~nm}$ (MEH-s X). The hypsochromic shift found for both, absorption and emission spectra, was associated to the postulated formation of shorter oligomeric fragments upon dilution of the initial polymer solution. This fact is in agreement with the decrease of the QY and increase of the fluorescent lifetime upon polymer dilution (Table S1), following similar trends to those found for PPV oligomers, where shorter conjugation domains present longer emission lifetime. ${ }^{[14]}$

Confirmation of the fragmentation process was obtained by chemical means. ${ }^{1} \mathrm{H}-\mathrm{NMR}$ spectra of both samples (Figure S1) showed new signals in the 7.0-8.0 ppm regions, mainly associated to modifications of the vinylene moieties in the new-formed species. The weak signals at 9.77 and $10.42 \mathrm{ppm}$ were attributed to the formation of carbonyl moieties, whose presence was also supported by new peaks in FT-IR spectra (Figure S2): $1730 \mathrm{~cm}^{-1}, \mathrm{C}=\mathrm{O}$ 
stretching of aromatic aldehydes, $1680 \mathrm{~cm}^{-1}, \mathrm{C}=\mathrm{O}$ stretching of carboxylic groups. The incorporation of these functionalities in MEH-PPV by different chemical means has already been shown to take place through oxidative cleavage, ${ }^{[18]}$ which in the case of conjugated systems can be induced by $\mathrm{HOCl} .{ }^{[21-23]}$ No relevant intensity modifications were observed for $\mathrm{C}-\mathrm{H}$ stretching of the methoxy and alkoxy groups $\left(1037 \mathrm{~cm}^{-1}\right)$ and to the aromatic $\mathrm{C}=\mathrm{C}$ stretching $\left(1502 \mathrm{~cm}^{-1}\right)$ peaks, confirming that the sonication induced mainly chemical changes to the vinylene groups. X-Ray photoelectron spectroscopy (XPS) of the samples revealed a concomitant increase of the oxygen (from the $11.97 \%$ of the parent polymer to $21.16 \%$ for MEH-s X) and chlorine (from the $0.61 \%$ to $2.16 \%$ ), along with a decrease of the relative carbon amount (from $87.42 \%$ to $76.67 \%$ ), as the polymer concentration of the initial solution decreased, indicating a higher degree of fragmentation (Figure S3). Elemental analysis of the treated samples showed a similar trend, with the relative carbon amount decreasing (MEH-s $4 \mathrm{x}, 70.0 \%$; MEH-s X, 63.7\%) as the concentration of the initial polymer solution diminishes (Table S2).

FT-Raman spectra recorded for MEH-s X and MEH-s 4X samples (Figure S4) were quite similar, with the presence of an intense band located around $1580-1590 \mathrm{~cm}^{-1}$, which was ascribed to $\mathrm{C}-\mathrm{C}$ stretching vibration of the phenyl rings and a weaker band at a longer wavenumber, approximately at $1625 \mathrm{~cm}^{-1}$, arising from the $\mathrm{C}=\mathrm{C}$ stretching of the vinyl group. Two trends were found along the samples regarding these two aforementioned bands: $a$ ) there is a gradual upshift of the whole spectrum upon going from MEH-PPV to MEH-s $4 \mathrm{X}$ and MEH-s X, with a shift of the most intense band from $1581 \mathrm{~cm}^{-1}$ (MEH-PPV) to $1602 \mathrm{~cm}^{-1}$ (MEH-s X), and $b$ ) the intensity ratio $\mathrm{I} \sim 1625 / \mathrm{I} \sim 1585$ increases as the concentration of the starting polymer solution diminishes. These exact same trends were reported for a series of PPV derivatives, with the progressive decreasing of the chain length, ${ }^{[27-29]}$ and can be easily 
tracked down theoretically (Figures S5-S7). Therefore, sonication of the sample creates shorter PPV fragments and/or disruption of effective conjugation length due to a direct attack of the vinyl group. These two effects would appear similarly in Raman spectroscopy, since theoretical DFT calculations predict almost superimposable spectra for a 2PPV fragment and a 4PPV in which the central vinylene group has been attacked by the acidified solution (4PPVCl). Therefore, these results highlight that fragmentation of the samples, either by conjugation disruption or by selective chain cleavage, is more efficient in less concentrated samples.

The formation of oligomeric units of lower molecular weight than the untreated MEHPPV was supported by diffusion-ordered NMR spectroscopy (DOSY) and gel permeation chromatography (GPC). Both sonicated samples, showed higher diffusion coefficient parameters than for the untreated MEH-PPV (Figure S8), indicating a lower molecular weight for the obtained compounds. Accordingly, only species of lower number average molecular weight $(\mathrm{Mn})$ than untreated MEH-PPV $(\mathrm{Mn}=40.000-70.000)$ were detected by GPC for the MEH-s X and MEH-s 4X samples, confirming the sonication produces shorter oligomers rather than only partial saturation of the initial polymer (Table S3).

Finally, and to demonstrate that this process was not restricted to a few specific cases, we decided to investigate the behaviour, upon sonication, of mixtures prepared from $\mathrm{MEH}$ PPV solutions with concentrations of $4 \mathrm{mM}(2 \mathrm{x}), 6 \mathrm{mM}(3 \mathrm{x})$ and $12 \mathrm{mM}(6 \mathrm{x})$. Interestingly, sonicating these solutions with the same conditions as above, novel absorption and emission bands appeared between those obtained from the non-treated polymer and the lowest concentrated sonicated $(2 \mathrm{mM})$ solution. In this way, we fully demonstrated how by simply changing the initial concentration of the MEH-PPV polymer, we were able to fine tune the optical properties along the whole visible region of the formed oligomeric species (Figure S9). 
Once the fragmentation and the electronic disruption was confirmed, further experiments were carried out to study the reaction mechanism. As initially mentioned, polymer fragmentation could be attributed to the reactivity of the polymer with different species formed along the sonication process, mainly chlorine radicals, produced directly from the sonication of $\mathrm{CHCl}_{3}$, and $\mathrm{HOCl}$, obtained as secondary species from the sonication of $\mathrm{CHCl}_{3} / \mathrm{H}_{2} \mathrm{O}$ mixture. First blank experiments consisted of $a$ ) the sonication of a MEH-PPV chloroform solution in the absence of water, where only chlorine radicals (and no $\mathrm{HOCl}$ ) are formed according to previous literature, ${ }^{[30]}$ and $b$ ) the replacement of $\mathrm{CHCl}_{3}$ by toluene or $\mathrm{CH}_{2} \mathrm{Cl}_{2}$ in the nanoemulsion preparation, where chlorine radicals production is avoided or much less favored and no $\mathrm{HOCl}$ is formed. Interestingly, no relevant modifications of the absorption and emission spectra were found (Figures S10 and S11), pointing out the crucial role played by the $\mathrm{HOCl}$ in the fragmentation process. In fact, the relevance of $\mathrm{HOCl}$ is not surprising at all as it has already been shown to produce the cleavage of linear conjugated systems with the consequent formation of carbonyl moieties. ${ }^{[21-23]}$ To confirm this, a $\mathrm{CH}_{2} \mathrm{Cl}_{2}$ solution of MEH-PPV ( $8 \mathrm{mM})$ was treated with a $\mathrm{NaOCl} / \mathrm{HOCl}$ acidified aqueous solution $(\mathrm{pH}=6)$, without sonication. Also in this case, a progressive hypsochromic shift of the main absorption and emission bands over time was observed (Figure S12). ${ }^{1} \mathrm{H}-\mathrm{NMR}$ spectral changes of the HOCl-treated samples measured at selected reaction times $\mathrm{t}$ (HA-t, Figure S13a) indicated the formation of new species resembling those obtained through sonication (MEH-s). Notably, also in the HOCl-treated $\mathrm{CH}_{2} \mathrm{Cl}_{2}$ solution of MEH-PPV (e.g. HA-21h, Figure S13b) the signals at 10.42 and $9.77 \mathrm{ppm}$, related to carbonyl moieties resulting from the cleavage of the conjugated system, were observed. The role of $\mathrm{HOCl}$ in the process was finally assessed upon: a) sonication of a $\mathrm{MEH}-\mathrm{PPV} \mathrm{CHCl}_{3}$ solution in a basic water solution $(\mathrm{NaOH}, 1 \mathrm{M})$, which is prompted to neutralize the formed $\mathrm{HOCl}$ (Figure S14) and $b$ ) 
sonication of MEH-PPV in a $\mathrm{CHCl}_{3} / \mathrm{H}_{2} \mathrm{O}$ solution in the presence of resorcinol as a $\mathrm{HOCl}$ scavenger $^{[31]}$ (Figure S15). In all cases a dramatic reduction of the blue-shift was detected as the formation and/or activity of $\mathrm{HOCl}$ was hindered.

To simultaneously achieve the optical tuning and the formation of nanoparticles (CNPs) via a modified miniemulsion method, ${ }^{[32]}$ polymer solutions of six different concentrations, named $2 \mathrm{mM}(\mathrm{X}), 4 \mathrm{mM}(2 \mathrm{X}), 6 \mathrm{mM}(3 \mathrm{X}), 8 \mathrm{mM}(4 \mathrm{X}), 12 \mathrm{mM}(6 \mathrm{X})$ and 20 $\mathrm{mM}(10 \mathrm{X})$, were sonicated again in Milli- $\mathrm{Q}^{\circledR}$ water but now in the presence of a surfactant (AOT, $0.1 \mathrm{wt} . \%)$ that stabilizes the chloroform-in-water nanoemulsion. Similarly to what observed for the non-containing surfactant mixtures, the spectroscopic UV-Vis characterization of the sonicated samples showed a progressive hypsochromic shift of the main absorption bands of the CNPs (from $\lambda_{\max }=476 \mathrm{~nm}$ to $306 \mathrm{~nm}$ ) as the concentration of polymer in the initial $\mathrm{CHCl}_{3}$ solution decreases (Figure 2a).

The related blue-shift correlation of the emission bands (from $\lambda_{\max }=597 \mathrm{~nm}$ to 481 $\mathrm{nm}$ ) with the initial polymer concentration was also found for the CNPs (Figure 2b). TEM and SEM (Figure S16) images showed the expected formation of nanoparticles for all the sonicated mixtures, with dimensions ranging from $62 \pm 7 \mathrm{~nm}$ to $146 \pm 23 \mathrm{~nm}$. Notably, minor impurities of micro/nanosized cubic crystals, confirmed to be $\mathrm{NaCl}$ by $\mathrm{EDX}$ analysis, were also found (Figure S17). These crystals, formed after sonication even in the absence of the polymer (Figure S18) and the surfactant, were most likely obtained by the recombination of chlorine species and traces of sodium found in the water as well as in the walls of the vial, and/or by decomposition of $\mathrm{NaOCl}^{[33]}$ formed upon recombination of $\mathrm{HOCl}$ and the sodium ions traces. The nanoparticles showed red shift emission properties respect to the fragmented species obtained upon sonication of the mixtures of same concentration, but without surfactant (MEH-s). This red shift can be explained on the basis of different intermolecular 
interactions between the oligomeric chains induced by the close packing present in the nanoparticles, as already described for several conjugated polymer nanoparticles, ${ }^{[34-35]}$ rather than to the formation of different oligomeric species. The interchain interactions of the polymer within the CNPs favour the overlap between $\pi$ orbitals, enlarging the conjugation, increasing the exciton diffusion and thus yielding lower energy emission. ${ }^{[34-35]}$ This was supported by FT-Raman spectroscopy (Figure S19). The recorded data of the CNPs samples indicate similar spectra profiles as those obtained for the non-structured oligomers. 
a)

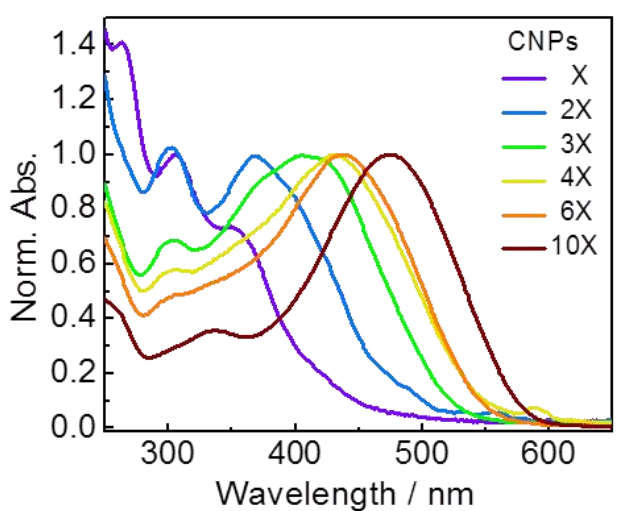

b)

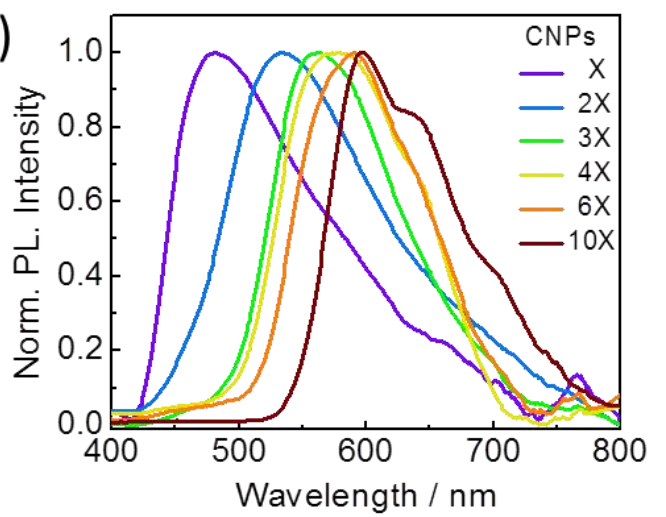

c)

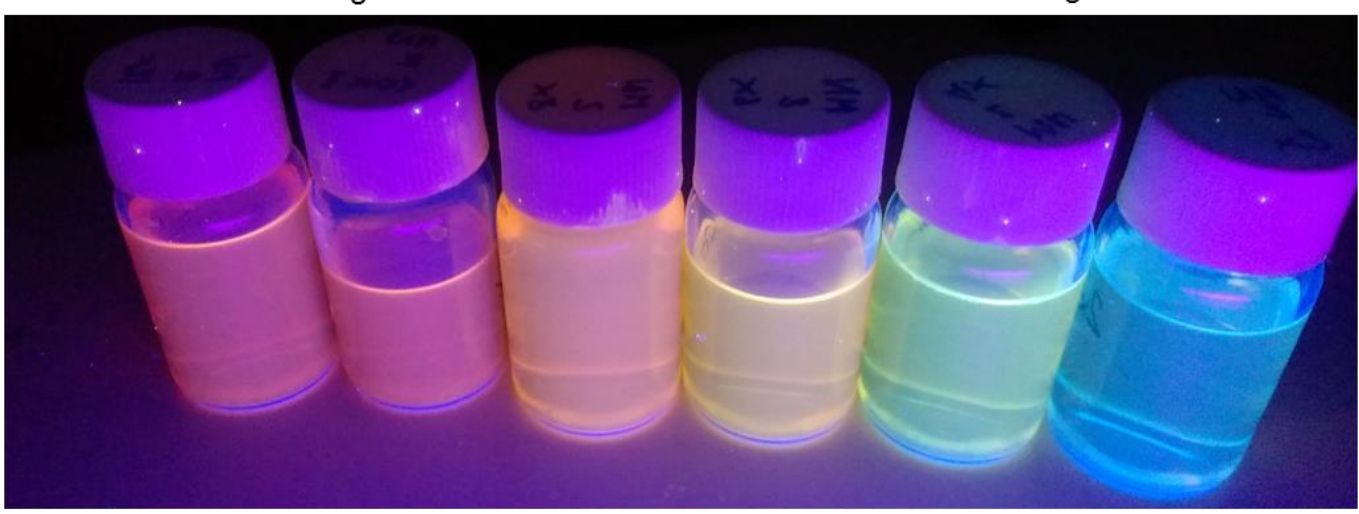

d)
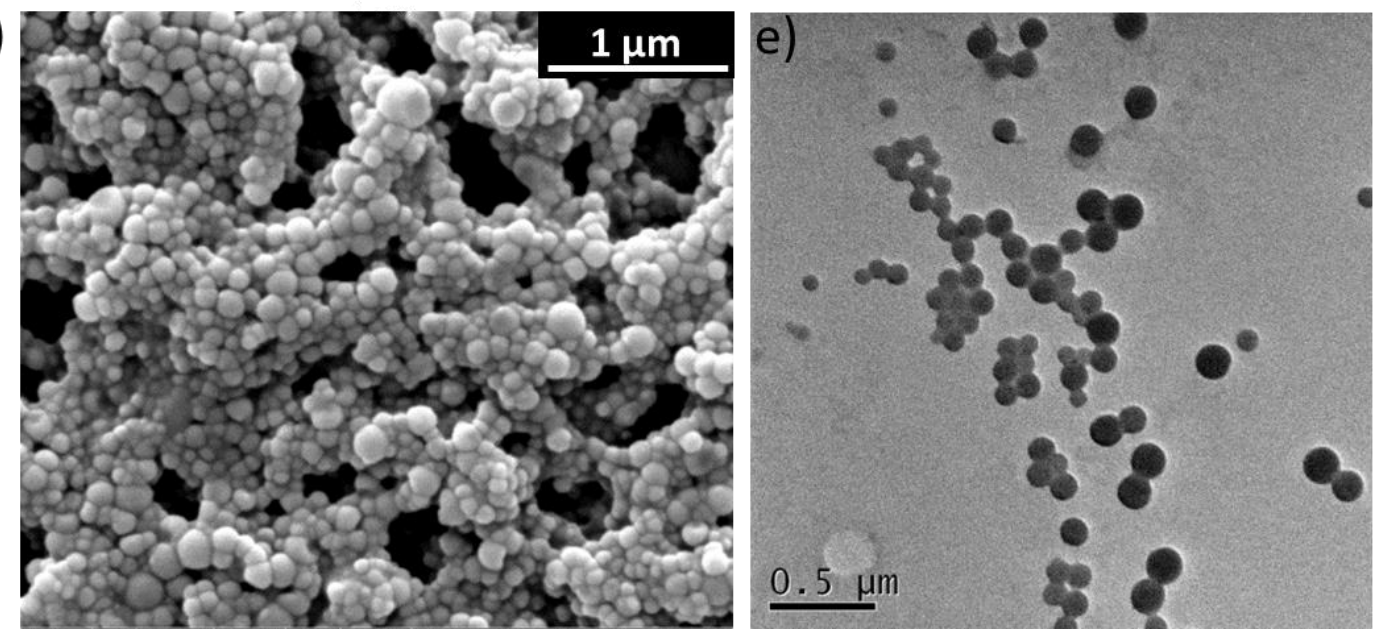

Figure 2. a) absorption, $b$ ) emission spectra and c) digital photograph of the series of CNPs_X-10X; $d$ ) SEM and $e$ ) TEM images of CNPs_4X suspensions.

Furthermore, CNPs_3X presented its most intense Raman band $\left(1592 \mathrm{~cm}^{-1}\right)$ upshifted respect to CNPs_6X $\left(1585 \mathrm{~cm}^{-1}\right)$, and a higher I $1625 / \mathrm{I} \sim 1585$ ratio, both features indicative 
of shorter conjugated chains as the initial polymeric concentration decreases; that is, also supporting fragmentation into oligomers in the nanoemulsion.

Finally, on the pursuit for functional materials, all CNPs suspensions (CNPs_X-10X) were used to form self-standing, flexible polyvinyl alcohol (PVA) films (CNPs_X10X@PVA), through drop casting (see supporting information).
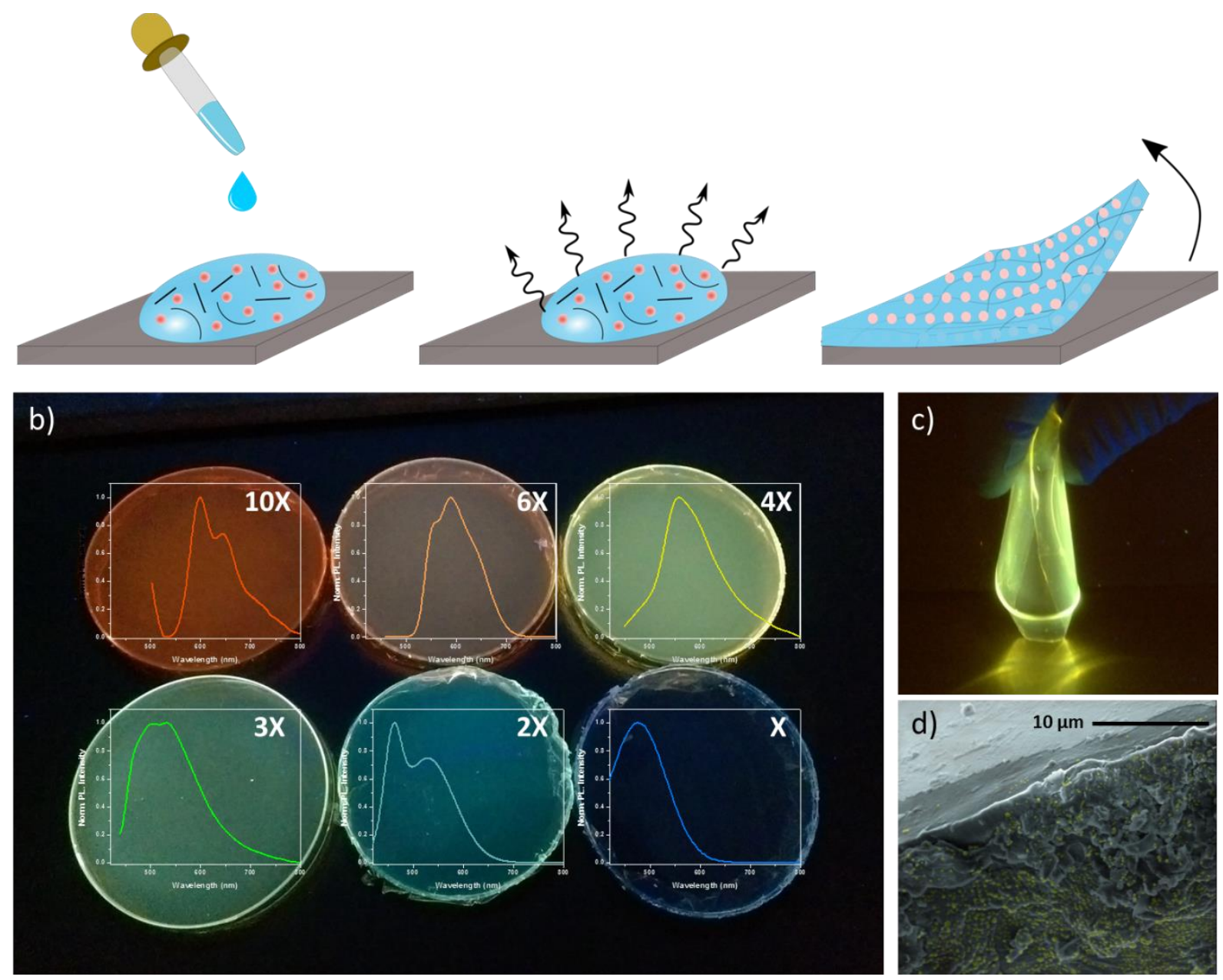

Figure 3. a) scheme of drop-casting process of the CNPs_X-10X suspensions leading to the formation of the PVA films after $\mathrm{H}_{2} \mathrm{O}$ evaporation; $b$ ) digital photographs of CNPs_X10X@PVA films and corresponding emission spectra; $c$ ) digital photograph of bended CNPs_4X@PVA fluorescent film showing its flexibility; $d$ ) SEM picture of CNPs_4X@PVA film cross section.

As can be seen, the absorption (Figure S20) and emission (Figure 3) properties of the PVA films resemble those of the CNPs suspensions, indicating that the spectral features of the 
nanoparticles are transferred to a polymeric functional material. Only minor additional bands appear in the spectra of the films by comparison to the colloidal nanoparticles suspensions, most likely due partial loss of NPs structure and/or mutual diffusion of MEH-PPV and PVA chains.

In summary, the reactive species $\mathrm{HOCl}$ generated upon sonication of a chloroform/water mixture is used to fragment the MEH-PPV polymer, with a degree of fragmentation tuned by its concentration in the initial organic solution; at lower polymer concentrations there are less polymer chains to be cleaved and therefore smaller fragments than for the more concentrated solutions are obtained over the same sonication time. Such fragmentation accounts for the systematic tuning of the optical properties. Though, worth to mention, previous synthetic studies revealed that optical shifts are no longer effective for oligomers with 10 units or more ${ }^{[14]}$ considerably smaller than those obtained in this work. So to fully explain our experimental observation, beyond complete fragmentation, partial saturation of vinylene moieties without fragmentation, ${ }^{[18]}$ must also be claimed. Finally, when the sonication process is done in the presence of surfactants, the nanoemulsion is stabilized resulting in the final formation of nanoparticles with tuned optical properties. Thanks to stability of such nanoparticles, these could be further integrated into polymeric matrices leading to the obtaining of self-standing and flexible fluorescent films. 


\section{Experimental Section}

Other experimental details (methods, characterization techniques and supporting figures, tables and schemes) are reported in the supporting information (SI).

\section{Supporting Information}

Supporting Information is available from the Wiley Online Library or from the author.

\section{Acknowledgments}

This work was supported by project MAT2015-70615-R from the Spanish Government and by FEDER funds. ICN2 acknowledges support from the Severo Ochoa Program (MINECO, Grant SEV-2013-0295). C.B. would like to thank ICN2 Severo Ochoa program and ICN2 for the grant. Funded by the CERCA Programme/Generalitat de Catalunya.

Received: ((will be filled in by the editorial staff))

Revised: ((will be filled in by the editorial staff))

Published online: ((will be filled in by the editorial staff))

\section{References.}


[1] T. Kietzke, D. Neher, K. Landfester, R. Montenegro, R. Güntner, U. Scherf, Nat. Mater. 2003, 2, 408.

[2] J. Wei, S. Xiong, Y. Bai, P. Jia, J. Ma, L. Xuehong, Sol. Energy Mater. Sol. Cells 2012, 99, 141.

[3] E. Park, T. Erdem, V. Ibrahimova, S. Nizamoglu, H. V. Demir, D. Tuncel, ACS Nano 2011, 5,4 .

[4] H. Piwoński, T. Michinobu, S. Habuchi, Nat. Comm. 2017, 8, 15256.

[5] K. Schwarz, S. Farley, T. Smith, K. P. Ghiggino, Nanoscale, 2015, 7, 47.

[6] X. Shen, F. He, J. Wu, G. Q. Xu, S. Q. Yao, Q.-H. Xu, Langmuir 2011, 27, 5.

[7] A. J. Cadby, S. H. Tolbert, J. Phys. Chem. B 2005, 109, 38.

[8] S. Quan, F. Teng, Z. Xu, L. Qian, Y. Hou, Y. Wang, X. Xu, Eur. Poyml. J. 2006, 42, 1.

[9] A. Köhler, S. T. Hoffmann, H. Bassler, J. Am. Chem. Soc. 2012, 134, 28.

[10] Y-S Huang, J. Gierschner, J. P. Schmidtke, R.H. Friend, D. Beljonne, Phys. Rev. B 2011, $84,20$.

[11] K.-T. Tung, C.-C. Chen, P. Lee, Y.-W. Liu, T.-M. Hong, K. C. Hwang, J. H. Hsu, J. D. White, A. C.-M. Yang, ACS Nano 2011, 5, 9.

[12] J. A. Schneider, A. Dadvand, W. Wen, D. F. Perepichka, Macromolecules 2013, 46, 23.

[13] T. Matsumoto, K. Tanaka, Y. Chujo, Macromolecules 2015, 48, 5.

[14] A. J. Tilley, S. M. Danczak, C. Browne, T. Young, T. Tan, K. P. Ghiggino, T. A. Smith, J. White, J. Org. Chem. 2011, 76, 9.

[15] M. Franke, P. Braeutigam, Z. Wu, Y. Ren, B. Ondruschka, Ultrason. Sonochem. 2011, 18,4 .

[16] Z.-L. Wu, B. Ondruschka and P. Bräutigam, Chem. Eng. Technol., 2007, 30, 5.

[17] D. Bondarev, O. Trhlíková, J. Sedláček, J. Vohlídal, Polym. Degrad. Stab 2014, 110.

[18] E. S. Bronze-Uhle, A. Batagin-Neto, F. C. Lavarda, C. F. O. Graeff, J. Appl. Phys. 2011, 110,7 .

[19] H.Wang, X. Tao, E. Newton, Polym. Int. 2004, 53, 1279.

[20] B. Bao, N. Tao, D. Yang, L. Yuwen, L. Weng, Q. Fan, W. Huang, L. Wang, Chem. Commun. 2013, 49, 90.

[21] O. Sommerburg, C.-D. Langhans, J. Arnhold, M. Leichsenring, C. Salerno, C. Crifò, G. F. Hoffmann, K.-M. Debatin, W. G. Siems, Free Radical Biol. Med. 2003, 35, 11

[22] D. Maitra, J. Byun, P. R. Andreana, I. Abdulhamid, M. P. Diamond, G. M. Saed, S. Pennathur, H. M. Abu-Soud, Free Radical Biol. Med. 2011, 51, 2.

[23] S. Pennathur, D. Maitra, J. Byun, I. Sliskovic, I. Abdulhamid, G. M. Saed, M. P. Diamond, H. M. Abu-Soud, Free Radical Biol. Med. 2010, 49, 2.

[24] C. J. Collison, L. J. Rothberg, V. Treemaneekarn, L. Yi, Macromolecules 2001, 34, 7.

[25] J. H. Burroughes, D. D. C. Bradley, A. R. Brown, R. N. Marks, K. Mackay, R. H. Friend, P. L. Burns, A. B. Holmes, Nature 1990, 347, 539. 
[26] Y. Pang, M. Samoc, P. N. Prasad, J. Chem. Phys. 1991, 94, 8.

[27] B. Tian, G. Zerbi, K. Müllen, J. Chem. Phys. 1991, 95, 1.

[28] I. Orion, J. P. Buisson, S. Lefrant, Phys. Rev. B 1998, 57, 12.

[29] E. Mulazzi, A. Ripamonti, J. Wery, B. Dulieu, S. Lefrant, Phys. Rev. 1999, 60, 24.

[30] A. Henglein, Ch.-H. Fischer, Ber. Bunsenges. Phys. Chem. 1984, 88, 12.

[31] M. Özyürek, B. Bekdeşer, K. Güçlü, R. Apak, Anal. Chem. 2012, 84, 21.

[32] K. Landfester, R. Montenegro, U. Scherf, R. Güntner, U. Asawapirom, S. Patil, D. Neher, T. Kietzke Adv. Mater. 2002, 14, 9.

[33] J. March, B. Simonet, Talanta 2007, 73, 2.

[34] J. K. Grey, D. Y. Kim, B. C. Norris, W. L. Miller, P. F. Barbara, J. Phys. Chem. $B$ 2006, $110,51$.

[35] C. Wu, J. McNeill, Langmuir 2008, 24, 11. 


\section{Table of content:}

One-pot sonication-induced fragmentation and nanostructuration of seminconductive polymers is used to obtain conjugated polymer nanoparticles with fine-tuned absorption and emission properties in the whole visible spectral region. The sonication induces both breakage and structuration of the polymer chains, while the conjugation length and the corresponding optical properties are controlled by simply varying the polymer concentration of the initial solution.

Keywords: semiconducting polymers, fluorescent nanoparticles, sonochemistry, nanoemulsions, MEH-PPV

C. Bellacanzone, C. Roscini, * M. C. Ruiz Delgado, R. Ponce Ortiz, D. Ruiz-Molina*

Sonochemical synthesis of optically tunable conjugated polymer nanoparticles

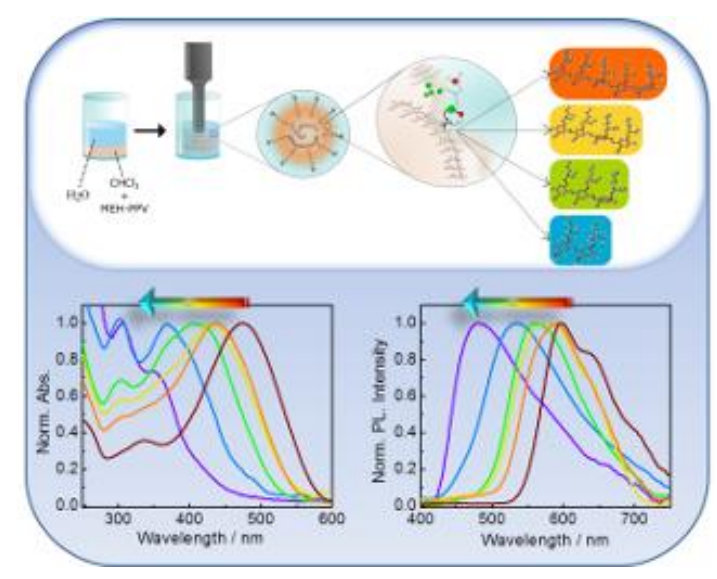

